\section{Schimmelpenning Syndrome: A Kind of Craniofacial Epidermal Nevus Associated with Cerebral and Ocular MR Imaging Abnormalities}

This letter focuses on Schimmelpenning syndrome (SS), a neurocutaneous disorder related to epidermal nevus syndromes and characterized by craniofacial nevus, neurologic anomalies, and ocular pathology.

We discuss clinical and MR imaging features of a 10-year-old boy, pointing out the etiopathologic substratum of this condition and the vascular origin of brain abnormalities; in our case, there is an unusual association of hemimacrocephaly and ipsilateral cerebral hemiatrophy.

Epidermal nevus syndrome is a rare phacomatosis characterized by epidermal nevi in association with neurologic disorders and ocular or skeletal anomalies; other organ systems may be involved with hamartomas. The term "epidermal nevus syndrome" does not indicate 1 disease but rather a heterogeneous group of neurocutaneous disorders with a distinct genetic profile and a similar cutaneous phenotype. ${ }^{1}$ These conditions may differ from one another either by type and site of nevi or by distinctive clinical correlations.

SS represents the best definition for a craniofacial sebaceus nevus associated with difficult seizures, mental retardation, and ocular lesions, such as coloboma or epibulbar lipodermoid; skull deformity is usually present. Cerebral manifestations include developmental anomalies, vascular lesions, tumors, and tumorlike conditions, which are all generally ipsilateral to the nevi. ${ }^{2,3}$

The classic form of SS is characterized by craniofacial hemihypertrophy, hemimegalencephaly, and pachygyria-polymicrogyria. The reported case of a large epidermal nevus of the head and neck differs from the classic form and consists of cerebral hemiatrophy with normal cortical organization, vascular anomalies, and giant Virchow-Robin spaces (VRSs).

The pathogenesis and clinical expression of SS and correlate syndromes are based on genomic mosaicism, in which $\geq 2$ genotypical different clones of the same specific cell line coexist in a single individual.

Epidermal and pigmentary cells originate early in the neural crest and, after proliferation, migrate to the periphery of the embryo. In neurocutaneous disorders, a somatic mutation at this time originates an altered cellular clone that will be phenotypically represented along the so-called lines of Blaschko (Fig 1). These lines indicate the pathway of embryonic ectoderm and are clinically apparent when recognizable from surrounding normal cells, thus representing the cutaneous expression of mosaicism. When genetic mutations occur early and their products do not cause the death of the embryo because of involvement of only some cell lineages, by the lethal gene theory, ${ }^{4}$ skin and the embryogenetically contiguous central nervous system will both be damaged.

The neurologic abnormalities are strictly related to the timing of these events. If mutation occurs during neuronal migration or cortical organization, there will be brain malformations; whereas if it happens later, atrophy and vascular anomalies will be more evident.

In our patient, unilateral hypoplasia of the arterial system, Sylvian stenosis, and ischemic changes in the temporal lobe (Figs 2 and 3) should refer to a malformative vasculopathy that occurred late in utero or at least after brain gyration and seriously reduced hemispheric perfusion. In fact, patients with SS are at risk of vascular anomalies, as reported in approximately one-third of published cases; among these anomalies are aortic coarctation and aneurysm, and renal artery and carotid stenosis. ${ }^{5}$

In conclusion, our patient exhibited typical features of SS with craniofacial epidermal nevus, ipsilateral cerebral abnormalities, difficult epilepsy, mental retardation, and ocular pathology; such characteristics give us the possibility of distinguishing this condition from the other related disorders. The relevance of this case is in the unusual association of hemimacrocrania and ipsilateral cerebral atrophy; moreover, brain abnormalities are the result of an in utero vasculopathy and do not include cortical dysplasia as in most of the previous reports.

\section{References}

1. Happle R. Epidermal nevus syndrome. Semin Dermatol 1995;14:111-2

2. Sugarman JL. Epidermal nevus syndrome. Semin Cutan Med Surg 2007;26:221-30
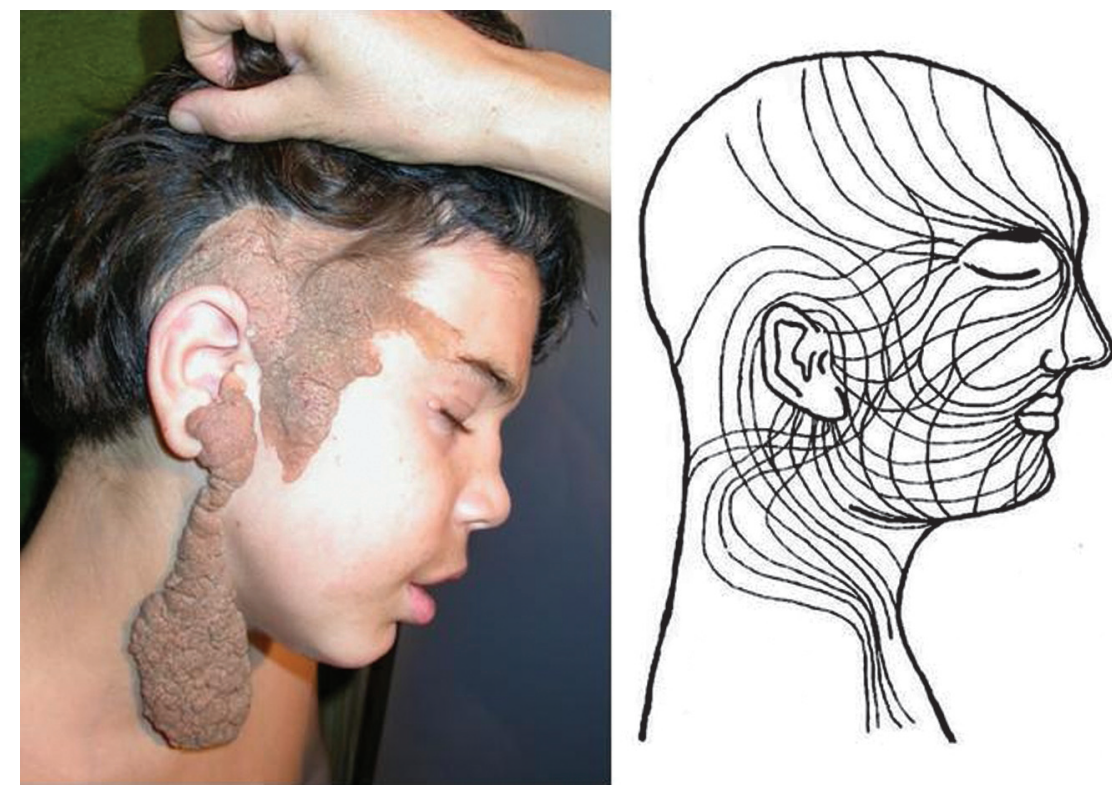

Fig 1. A, Patient at 10 years old with a large, tumefactive, and hyperkeratotic epidermal nevus involving the skull, face, and neck. $B$, Note lines of Blaschko on the head and neck. Modified from Moss et al. ${ }^{4}$ 


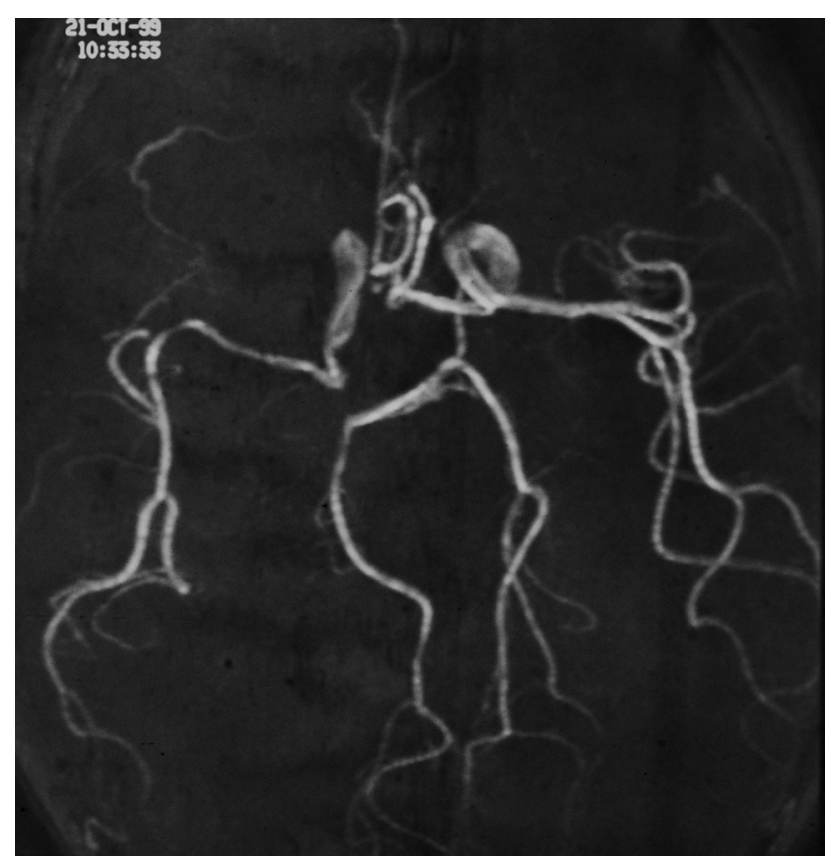

Fig 2. MR angiography of the patient at 2 years old shows a hypoplastic right siphon and middle cerebral artery, with a stenotic Sylvian bifurcation.
3. Zhang W, Simos PG, Ishibashi I, et al. Neuroimaging features of epidermal nevus syndrome. AJNR Am J Neuroradiol 2003;24:1468-70

4. Moss C, Larkins S, Stacey M, et al. Epidermal mosaicism and Blaschko's lines. J Med Genet 1993;30:752-55

5. Greene AK, Rogers GF, Mulliken JB. Schimmelpenning syndrome: an association with vascular anomalies. Cleft Palate Craniofac J 2007;44:208-15

C. Amato

Department of Neuroradiology

M. Elia

Department of Neurology

C. Schepis

Department of Dermatology

“Oasi Maria SS."-Research Institute (IRCCS)

Troina, Italy
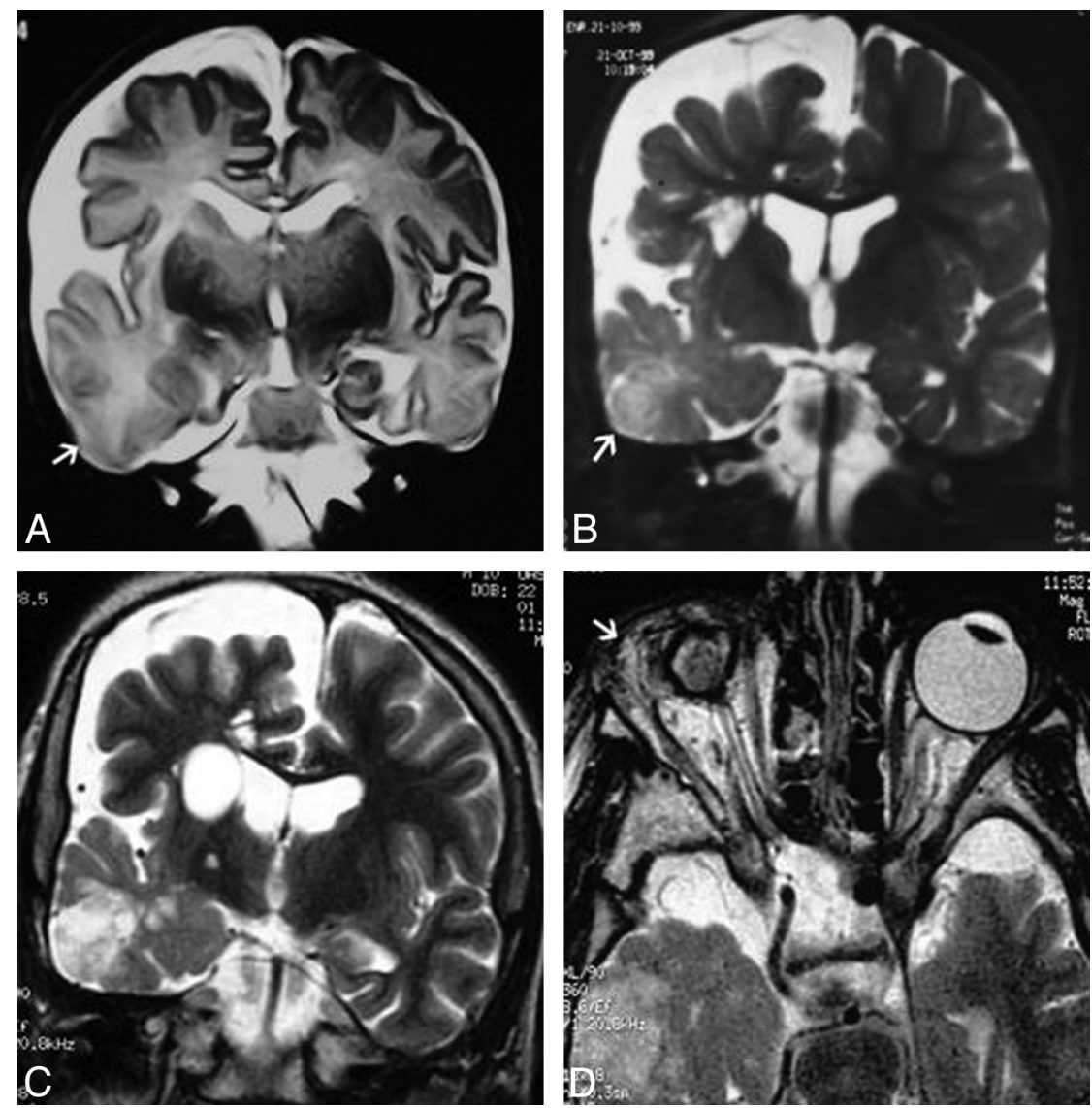

Fig 3. A, Coronal T2-weighted image of the patient at 20 days old shows a right cerebral hemiatrophy with cortical thinning and mild parenchymal change in the temporal lobe (arrow) On the same side, macrocrania and dilated liquoral spaces are present. B, Coronal T2-weighted image of the patient at 2 years old shows hypomyelination and an old infarct (arrow) in the right temporal lobe. Next to the ventricle, confluent and enlarged VRSs are recognizable. C, MR image of the patient at 10 years old. Coronal T2-weighted image shows, on the right side, stable cerebral hemiatrophy, a giant VRS next to the ventricle, and old ischemic changes within the hypomyelinated temporal lobe. $D$, High-resolution axial T2-weighted image shows a scleroatrophic right eyeball with a hypoplastic optic nerve. Lipodermoid tissue in the lacrimal fossa is present (arrow). 